\title{
Sœurs de même taille : Attachement d'une phrase relative aux syntagmes nominaux coordonnés
}

\section{Same-Size-Sisters: Relative clause attachment to coordinated noun phrases}

\author{
Antoine Hedier \\ Université de Paris \& LLF \\ Peijia Su \\ Université de Paris \& LLF \\ Barbara Hemforth \\ Université de Paris \& LLF
}

\begin{abstract}
Résumé
Dans deux expériences, chacune réalisée en français et en anglais, nous montrons que la préférence d'attachement d'une phrase relative à une coordination de syntagmes nominaux peut être modifiée par la longueur du premier conjoint. Alors qu'un attachement haut à la coordination est généralement préféré dans les deux langues, un attachement bas au second conjoint seulement devient plus acceptable pour les phrases dont le premier conjoint est plus long. Ces résultats sont compatibles avec le principe des sœurs de même taille (Fodor 1998, 2002) qui suggère qu'une préférence prosodique pour les unités de taille équilibrée s'applique également au traitement du langage écrit, selon l'hypothèse de la prosodie implicite.
\end{abstract}

Mots-clés

Ambiguïté structurelle, attachement des phrases relatives, prosodie implicite

\begin{abstract}
In two experiments, each run in French and in English, we show that the length of the first conjunct can modify the attachment preference of a relative clause to two coordinated noun phrases. While high attachment to the coordination of the noun phrases is generally preferred across languages, low attachment to only the second conjunct becomes more acceptable for sentences with a longer first conjunct. These results are compatible with the Same-Size-Sisters principle (Fodor 1998, 2002), which suggests that a prosodic preference for units of balanced size also applies to the processing of written language via the Implicit Prosody Hypothesis.
\end{abstract}

Keywords

Structural ambiguity, relative clause attachment, implicit prosody 


\section{INTRODUCTION}

Les langues naturelles sont souvent sujettes à l'ambiguité, mais les locuteurs en sont rarement conscients. Généralement, ils choisissent l'une des interprétations possibles d'un mot, d'une phrase ou d'un syntagme par une combinaison d'heuristiques basée sur la fréquence de mots ou de constructions, la complexité de la représentation syntaxique, sémantique ou pragmatique, ou d'autres facteurs liés au contexte linguistique. En utilisant des méthodes expérimentales issues de la psychologie cognitive, un des objectifs de la linguistique expérimentale (et plus généralement de la psycholinguistique) est de définir les contraintes et préférences qui rendent les phrases plus ou moins acceptables ou plus ou moins faciles à traiter dans une langue et à déterminer les préférences d'interprétation pour le cas d'ambiguïté (Hemforth 2013; Hemforth et al. 2014). À ce propos, les ambiguités d'attachement d'un constituant à la structure de la phrase en cours de construction ont été beaucoup étudiées en linguistique expérimentale (voir, p. ex., Bard \& Aylett 1999 ; Pynte \& Colonna 2000 ; Bard et al. 2000 ; Hemforth et al. 2015).

\subsection{Clôture Tardive}

L'ambiguïté d'attachement ne peut faire abstraction du célèbre modèle de traitement des phrases à effet «Garden-Path » (Frazier 1987), caractérisé par le principe de l'Attachement Minimal (angl. Minimal Attachment, Frazier 1987). Celui-ci se base sur le fait que l'analyseur syntaxique préfère la structure la plus simple. Une deuxième stratégie de traitement est la Clôture Tardive (angl. Late Closure, Frazier 1978 ; Frazier \& Fodor 1978), qui suppose que le dernier élément apparaissant dans la phrase en cours de traitement tend à s'attacher au dernier syntagme analysé (alors au syntagme le plus proche). Ces préférences sont expliquées par un avantage d'efficacité et de coût cognitif moindre grâce à une économie de temps de computation réalisée par un attachement structurellement moins complexe et plus local (Wanner 1980 ; Fodor \& Frazier 1980).

Ces deux stratégies, faisant partie intégrante du traitement du langage humain, sont supposées s'appliquer à toutes les langues, éventuellement en interaction avec leurs propriétés spécifiques. Cela signifie que les différences qu'on peut observer dans la préférence pour une certaine interprétation d'une ambiguiité structurelle devraient trouver une justification dans les différences liées aux propriétés sousjacentes de la langue.

Tant qu'une telle différence n'a pas été identifiée, les mêmes schémas de préférence sont prédits. Ainsi, l'hypothèse d'une préférence universelle pour la Clôture Tardive a été remise en cause par un grand nombre d'études sur l'attachement des phrases relatives. Par exemple, dans les exemples (1a-d), la phrase relative peut être attachée à un syntagme nominal $(\mathrm{SN})$ plus distant et plus haut dans la structure syntaxique de la phrase (SN1, figure 1a), ou bien à un SN plus proche et plus bas (SN2, figure 1b). Le principe de l'Attachement Minimal ne s'appliquant pas dans ce cas (les deux attachements ne se distinguent pas en termes de leur complexité), la Clôture Tardive prédit une préférence pour un attachement bas ou local, ce qui est confirmé par la plupart des études en anglais (Cuetos \& Mitchell 1988). Cependant, une préférence pour l'attachement haut (figure 1a), c'est-à-dire une Clôture Anticipée (angl. Early Closure) a été trouvée pour l'attachement des 
phrases relatives à un syntagme nominal complexe dans beaucoup de langues comme l'espagnol (Cuetos \& Mitchell 1988), le français (Zagar, Pynte \& Rativeau 1997) ou l'allemand (Hemforth et al. 2000).

1. a) Français : le fils de l'enseignant qui ronfle

b) Anglais : the son of the teacher who snores

c) Allemand : der Sohn des Lehrers, der schnarcht

e) Espagnol : el hijo del maestro que ronca

Figure 1a. Attachement haut ${ }^{1}$
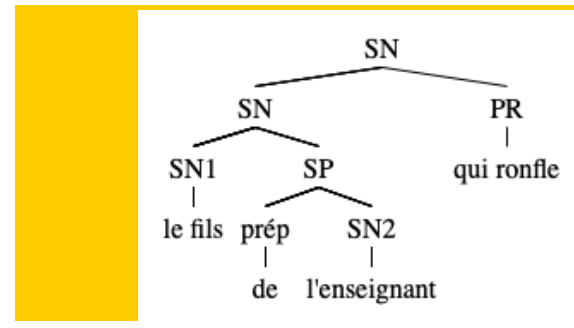

Pourtant, d'autres constructions d'ambiguïté d'attachement en espagnol ainsi que dans d'autres langues penchent encore pour un attachement local (p. ex. Igoa 1995 ; Gibson et al. 1996 ; Konieczny \& Hemforth 2000, pour l'attachement des syntagmes prépositionnels). De ce fait, l'attachement d'une phrase relative à une structure avec deux SN, comme dans les exemples (1a-d) ci-dessus, est souvent considéré comme une exception qui ne devrait pas remettre en cause l'universalité de la Clôture Tardive (Frazier \& Clifton 1996). Cependant, si la Clôture Tardive est toujours considérée comme un principe de traitement des phrases universellement applicable, les différences entre les langues dans les préférences d'attachement doivent s'expliquer par des différences dans les propriétés spécifiques des langues. Gibson et al. (1996) proposent que la tendance à l'attachement haut dans ces constructions vient du fait que le site d'attachement haut se trouve plus proche du prédicat. Cette contrainte de Proximité du Prédicat (angl. Predicate Proximity) servirait de contrepoids à la Clôture Tardive et serait peut-être plus importante dans certaines langues (éventuellement les langues avec un ordre des mots plus souple, comme l'espagnol par rapport à l'anglais). Plus récemment, les différences dans les préférences d'attachement à travers les langues ont été partiellement expliquées par la présence d'une analyse syntaxique alternative pour les phrases relatives, les pseudo-relatives, qui existent notamment dans la plupart des langues romanes (p. ex. il l'entend qui ronfle; Grillo \& Costa 2014 ; Pozniak et al. 2019).

Fodor (1998) propose une analyse basée sur le modèle de «Machine à Saucisses » (angl. Sausage Machine, Frazier \& Fodor 1978) qui pourrait expliquer une clôture anticipée pour les phrases relatives. Elle observe que le choix entre la Clôture Anticipée/Tardive peut être associé à la taille et à la catégorie syntaxique du

Toutes les structures syntaxiques dans cet article sont fortement simplifiées et ne servent qu'à des fins d'illustration. 
syntagme à attacher. En supposant que l'analyse syntaxique préfère diviser la phrase en 'paquets' de même taille, pour un syntagme comme Le fils de l'enseignant qui ronfle toute la nuit, la division en paquets pourrait entraîner une fermeture après la séquence l'enseignant, afin d'équilibrer la taille des paquets. Cependant, la préférence pour la Clôture Tardive dans cette construction en anglais a besoin d'être justifiée. Dans ses articles fondateurs, Fodor $(1998,2002)$ approfondit cette question liée à l'ambiguïté d'attachement, en accordant une importance à la prosodie.

\subsection{Le rôle de la prosodie dans la résolution des ambiguïtés structurelles}

La tradition des recherches expérimentales sur le traitement du langage se concentre sur les aspects syntaxiques et sémantiques en utilisant les matériels visuels à l'écrit et, par conséquent, les éventuelles contraintes prosodiques sont souvent ignorées. Fodor $(1998,2002)$ incorpore la prosodie dans la question du traitement du langage en supposant que les contours prosodiques sont calculés en parallèle avec la structure syntaxique. La structure prosodique influencera ensuite les décisions du système de traitement syntaxique, comme par exemple la préférence d'attachement d'un syntagme dans le cas d'une ambiguïté structurelle. Fodor affirme ainsi que les variations dans l'analyse syntaxique résultent en fait des différences prosodiques des langues. Le comportement différent de l'anglais sur l'attachement des phrases relatives au SN complexe pourrait s'expliquer par ses propriétés prosodiques exceptionnelles (Gilboy \& Sopena 1996).

Allant au-delà des études qui confirment l'influence du contour prosodique sur le traitement syntaxique à l'oral (p. ex. Beach 1991; Schafer et al. 2000 ; Michelas \& D'Imperio 2015), Fodor (2002) montre que, même en regardant les stimuli écrits, la psycholinguistique ne peut pas échapper à la prosodie. Avec l'Hypothèse de la Prosodie Implicite (angl. Implicit Prosody Hypothesis), Fodor propose que, même dans la lecture silencieuse, les frontières prosodiques sont calculées automatiquement et les contraintes prosodiques peuvent ainsi s'appliquer à l'analyse syntaxique, ce qui doit être pris en compte dans les expériences de lecture dans les études de traitement du langage.

En prenant en compte les aspects métriques de la prosodie, Fodor (1998: 302) propose le principe des "Sœurs de Même Taille " (angl. Same-Sized-Sisters, dorénavant SSS). Celui-ci se base sur la préférence prosodique pour les structures équilibrées à travers les langues, ce qui est confirmé dans les études phonologiques (p. ex. Cooper \& Paccia-Cooper 1980 ; Gee \& Grosjean 1983 ; Inoue \& Fodor 1995 ; Jun 2003 ; Kubozono 1993 ; Monnin \& Grosjean 1993). Selon le principe des SSS, le traitement prosodique préfère des syntagmes de même. Ainsi, dans le cas de l'ambiguïté d'attachement, un syntagme doit chercher un syntagme de taille comparable pour s'y joindre. Enfin, la contrainte prosodique des unités équilibrées est, selon Fodor $(1998,2002)$, le facteur sous-jacent de sa théorie antérieure appelée la « Machine à Saucisses ».

Le principe des SSS a été testé de manière assez approfondie sur l'attachement des phrases relatives à un SN complexe comme dans les exemples (1) ci-dessus (p. ex. en anglais, espagnol, français et allemand, cf. Hemforth et al. 2015) : les phrases relatives plus longues (p. ex. qui a ronflé [toute la nuit]) montrent une préférence plus forte pour un attachement haut, c'est-à-dire au SN complexe (figure 1a), alors 
que les phrases relatives plus courtes (p. ex. qui ronfle) montrent une préférence pour un attachement local ou bas, c'est-à-dire au deuxième $\mathrm{SN}$, qui est le plus proche (figure 1b) (cf. Hemforth et al. 2015 ; Pynte \& Colonna 2000).

Dans cet article, nous étudions les effets de longueur sur un autre type d'ambiguïté : l'attachement d'une phrase relative aux SN coordonnés (2). Ce phénomène, présent dans la plupart des langues, montre bien l'ambiguïté d'attachement de phrases relatives que nous avons décrite ci-dessus, mais il n'a pas encore été étudié dans la littérature psycholinguistique. En plus d'étudier une structure différente, nous voulons également examiner l'effet de longueur d'une manière différente. La plupart des travaux antérieurs ont fait varier la longueur du syntagme qui doit être attaché (p. ex. phrase relative, cf. Hemforth et al. 2015, ou syntagme prépositionnel, cf. Hemforth et al. 2013). Dans nos expériences, nous faisons varier la longueur des sites d'attachement potentiels.

2. les chiens et les chats qui jouent dans le jardin

Figure 2a. Attachement haut de la phrase relative
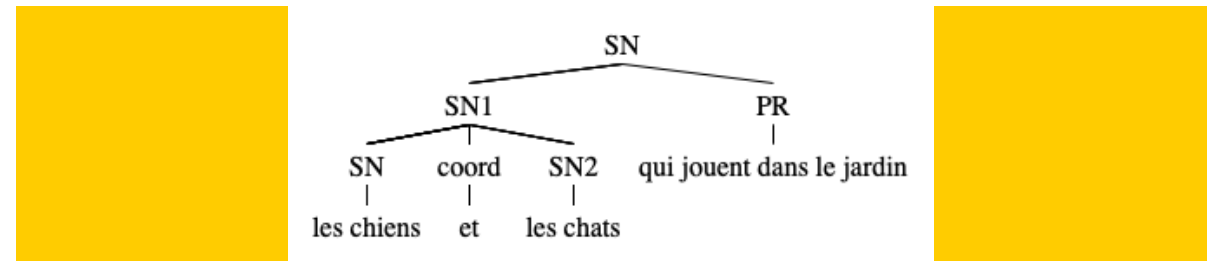

Figure $2 b$. Attachement bas de la phrase relative
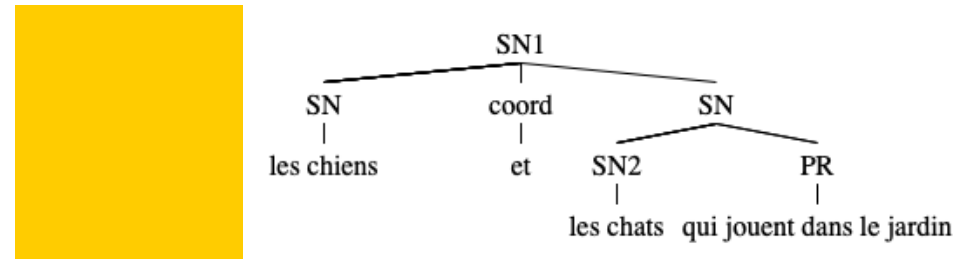

Dans nos matériels, la phrase relative peut potentiellement modifier la coordination dans son ensemble (attachement haut, SN1 les chiens et les chats, cf. figure 2a) ou seulement le SN le plus proche (attachement bas, SN2 les chats, cf. figure $2 \mathrm{~b}$ ). Le principe des SSS implique que les deux membres de la coordination doivent être équilibrés sur le plan prosodique. Cette contrainte d'équilibre prosodique est de moins en moins respectée quand la longueur de l'un des deux SN coordonnés augmente. Quand les deux SN sont de même taille, l'attachement local de la phrase relative (c'est-à-dire, au SN les chats) entraîne un fort déséquilibre avec une phrase relative assez longue qui s'attache à un $\mathrm{SN}$ assez court, de sorte que cela favoriserait l'attachement aux deux SN coordonnés. Par conséquent, la phrase relative devrait montrer une préférence pour un attachement haut. Cependant, plus le premier SN est long, plus la structure est équilibrée avec un attachement local. Dans 
nos expériences, nous avons fait varier la longueur du premier SN avec une version courte, comme en (3a), où le SN se compose d'un nom et d'un déterminant, une version moyenne, comme en (3b), où nous ajoutons un adjectif au $\mathrm{SN}$ de la version courte, et enfin une version longue, comme en $(3 \mathrm{c})$, où nous ajoutons un syntagme prépositionnel au SN de la version moyenne ${ }^{2}$.

3. a) Les chiens et les chats qui jouent dans le jardin vont être adoptés.

b) Les chiens blancs et les chats qui jouent dans le jardin vont être adoptés.

c) Les chiens blancs à taches noires et les chats qui jouent dans le jardin vont être adoptés.

Nous avons vu dans l'introduction que les préférences d'attachement des phrases relatives sont soumises à des variations à travers les langues. Même si nous supposons que l'hypothèse des SSS fonctionne de manière similaire dans toutes les langues, cela ne doit évidemment pas être considéré comme acquis. L'anglais et le français varient considérablement en ce qui concerne l'attachement des phrases relatives aux syntagmes nominaux complexes comme dans les exemples (1a-d), avec une préférence d'attachement bas en anglais et une préférence d'attachement haut en français (Cuetos \& Mitchell 1988 ; Zagar et al. 1997). Nous allons donc réaliser des versions étroitement parallèles de nos expériences dans les deux langues. De plus, vérifier une hypothèse dans une seule expérience n'est scientifiquement que peu convaincant. La force d'un résultat réside dans sa reproductibilité. Nous allons donc tester notre hypothèse à travers deux langues, mais aussi à travers deux paradigmes expérimentaux.

\section{PRemiere eXPerience}

Deux SN coordonnés suivis d'une phrase relative (Son ami et son frère qui ...) donnent lieu à deux interprétations possibles, ce qui rend cette structure de phrase ambiguë. Notre hypothèse est que, dans une situation où une phrase relative suit deux SN coordonnés, l'attachement de cette phrase relative peut être modulé selon la longueur du premier SN. Si la phrase relative se rapporte aux deux SN, l'attachement est dit «haut»; si la phrase relative se rapporte seulement au deuxième $\mathrm{SN}$, l'attachement est dit « bas ». Ces appellations d'attachement haut et bas viennent de la position de la phrase relative au sein des arbres syntaxiques dans les figures $2 \mathrm{a}$ et $2 \mathrm{~b}$. Si l'on prend l'exemple (3a) ci-dessus, l'interprétation qu'on obtient dans le cas d'un attachement haut est que les chiens et les chats jouent tous dans le jardin. Dans le cas d'un attachement bas, les chats sont les seuls à jouer dans le jardin.

\footnotetext{
'Ici nous faisons varier non seulement la longueur en nombre de syllabes, mais aussi la complexité syntaxique et la charge informationnelle (Almor 1999). Nous y reviendrons dans la discussion générale.
} 


\subsection{Protocole expérimental}

Pour tester l'hypothèse des SSS, nous utilisons le paradigme à double jugement d'acceptabilité sur une ambiguiité. Cette approche est différente du paradigme à choix binaire, généralement appliqué pour l'attachement des phrases relatives. Intuitivement, nous attendons une préférence forte pour l'attachement haut. Demander aux participants de choisir l'interprétation préférée donne une valeur binaire (attachement haut ou bas) et risque de ne pas saisir les variations plus fines de ce phénomène. C'est pourquoi, dans cette expérience, il est demandé aux participants de juger l'acceptabilité des deux interprétations.

\subsection{Matériel $^{3}$}

Nos items sont composés de deux SN coordonnés suivis d'une phrase relative puis d'un syntagme verbal. La phrase relative comporte toujours une extraction du sujet. Les phrases sont ensuite déclinées sous plusieurs variantes, appelées « conditions ». Le but est de changer minimalement ces phrases à travers les différentes conditions, afin de contrôler le plus précisément possible les différences entre les phrases et ainsi obtenir des comportements différents (contrastes minimaux). Ici, les différentes conditions dépendent de la longueur du premier $\mathrm{SN}^{4}$.

Dans la première condition, appelée dans le tableau 1 "Court», le SN est composé uniquement du nom et de son déterminant. Dans la deuxième condition, " Moyen », un adjectif est ajouté au SN. Dans la troisième condition, "Long », un adjectif et un syntagme prépositionnel ou deux adjectifs sont ajoutés au SN. Un total de 15 items a été créé, soit 45 phrases. Les items sont répartis sur trois listes de telle sorte qu'une seule des trois conditions de chaque item soit présentée dans chaque liste, mais que toutes les conditions soient présentées de manière équilibrée à travers les items (paradigme du carré latin). Une des trois listes est présentée à chaque participant de sorte que le nombre de participants par liste soit à peu près équilibré.

Tableau 1. Exemple d'un item français dans les trois conditions

\begin{tabular}{|l|l|}
\hline Court & Les assiettes et les fourchettes qui se trouvent sur l'étagère sont rayées. \\
\hline Moyen & $\begin{array}{l}\text { Les assiettes ovales et les fourchettes qui se trouvent sur l'étagère sont } \\
\text { rayées. }\end{array}$ \\
\hline Long & $\begin{array}{l}\text { Les assiettes à couscous ovales et les fourchettes qui se trouvent sur } \\
\text { l'étagère sont rayées. }\end{array}$ \\
\hline
\end{tabular}

Dans la version anglaise de l'expérience, les phrases conçues en français ont été traduites le plus fidèlement possible avec les mêmes variations de longueur.

\footnotetext{
Conformément aux directives générales pour la science ouverte, toutes les données brutes et les scripts sont anonymisées et accessibles à l'adresse suivante : https://osf.io/s46n3/.

* Voir la note 2 et la section dédiée à la discussion générale.
} 
Tableau 2. Exemple d'un item anglais dans les trois conditions

\begin{tabular}{|l|l|}
\hline Court & The plates and the forks that are on the shelf are scratched. \\
\hline Moyen & The oval plates and the forks that are on the shelf are scratched. \\
\hline Long & The oval couscous plates and the forks that are on the shelf are scratched. \\
\hline
\end{tabular}

Pour chaque expérience, l'utilisation de distracteurs est nécessaire pour éviter les effets d'habituation au phénomène étudié. Ces stimuli doivent répondre aux mêmes besoins du protocole expérimental, mais être fondamentalement différents de ceux utilisés dans l'expérience. 30 distracteurs de différents types ont été mélangés avec nos items expérimentaux. Les items et les distracteurs ont été présentés dans un ordre aléatoire différent pour chaque participant.

\subsection{Déroulement}

L'expérience était hébergée sur le site Ibex farm (Drummond et al. 2016, http://spellout.net/ibexfarm). Après avoir lu les instructions, avoir donné des informations sur leur âge, leur sexe, leur langue maternelle et leur consentement à participer volontairement à cette expérience, l'expérience commençait avec trois items d'entraînement. Pour chaque phrase, les participants devaient juger l'acceptabilité d'attachement de la phrase relative pour chaque interprétation. Par exemple, pour la phrase Les romans et les films qui parlent d'amour m'intéressent., la question suivante leur était posée : Laquelle des 2 descriptions convient le mieux ?.

Sur une échelle de 1 (pas du tout) à 5 (très bien), ils devaient juger l'acceptabilité de chacune des interprétations de la phrase : l'attachement haut (Les romans et les films parlent d'amour.) et l'attachement bas (Seuls les films parlent d'amour.).

Nous n'utilisons pas explicitement les termes 'phrase relative', 'syntagme nominal' ou encore 'attachement' dans les instructions. Nous posons des questions sur la compréhension globale de la phrase, afin de ne pas biaiser les réponses des participants. L'expérience durait approximativement 10 minutes.

\subsection{Participants}

Pour la version française de l'expérience, 31 adultes $^{5}$ (locuteurs natifs du français) ont été recrutés sur la plate-forme RISC (https://www.risc.cnrs.fr). La moyenne d'âge des participants est de 33.22 ans (minimum : 18 ; maximum : 76) pour 14 femmes et 17 hommes.

Pour la version anglaise de l'expérience, 29 adultes ${ }^{6}$ (locuteurs natifs de l'anglais) ont participé à cette expérience. Ils ont été recrutés via Amazon Mechanical Turk et ont reçu une compensation de 1.50\$. La moyenne d'âge des participants est de 37.76 ans (minimum : 24 ; maximum : 65) pour 8 femmes et 21 hommes.

\footnotetext{
s 2 personnes ont été exclues car le français n'était pas leur langue maternelle.

- 1 participant a été retiré, car il n'avait pas l'anglais comme langue maternelle.
} 


\subsection{Résultats}

En accord avec l'hypothèse des SSS et les résultats précédemment obtenus, nous attendons une préférence d'attachement modifiée graduellement selon la longueur du premier SN : plus le premier SN est long, plus on attend une préférence pour un attachement bas.

Pour l'analyse des données et ce tout au long de cet article, nous avons opté pour des analyses bayésiennes. Ces modèles ont certains avantages comparés aux analyses fréquentistes, plus traditionnelles dans les expériences en psycholinguistique. Un avantage pratique concerne le problème de convergence des modèles fréquentistes (modèles linéaires mixtes ou autres). Même s'il est fortement recommandé d'utiliser des modèles dits maximaux qui incluent la variation entre les participants et les items en ce qui concerne les intercepts ainsi que les pentes aléatoires des facteurs fixes (Barr et al. 2013), ces modèles ne peuvent pas toujours être calculés avec le nombre d'observations obtenu dans l'expérience, de sorte que des modèles inférieurs au maximum doivent être rapportés. Les modèles bayésiens sont systématiquement maximaux et prennent en compte les effets aléatoires (items et participants) ainsi que les pentes aléatoires individuelles.

Une différence fondamentale entre une analyse fréquentiste et une analyse bayésienne est la perspective sur l'hypothèse à tester : Une analyse fréquentiste tend à rejeter l'hypothèse nulle qui postule l'égalité entre deux paramètres statistiques (donc, l'absence d'effet), tandis que pour une analyse bayésienne, une simulation est mise en place pour calculer la probabilité de présence d'un effet. L'interprétation des analyses fréquentistes renvoie donc des résultats binaires, significatifs ou non (rejet de l'hypothèse nulle ou pas), tandis que les analyses bayésiennes renvoient des probabilités d'effet, ce qui permet de passer outre la contrainte de significativité statistique.

Pour toutes les analyses statistiques présentées dans cet article, nous avons utilisé le package brms dans le logiciel statistique $\mathrm{R}$ (Bürkner et al. 2018). Nous avons calculé des modèles avec un préalable (angl. prior) faiblement informatif par défaut et la famille cumulative adaptée aux données ordinales et ce avec 4 chaînes de 3000 itérations. Les modèles linéaires mixtes, souvent utilisés pour les jugements d'acceptabilité, ne sont pas adaptés à ce type de variable dépendante. Afin de nous assurer de la validité des modèles, nous avons vérifié la valeur Rhat pour chacun d'entre eux, qui était bien à 1 pour chaque analyse (ce qui signifie que le modèle n'est pas déficient).

Les résultats de l'expérience sur le français sont décrits dans la figure 3a. Ils montrent une nette préférence générale pour un attachement haut, mais aussi un effet de la longueur du premier conjoint. Plus le premier conjoint est long, plus les participants jugent acceptable l'interprétation de l'attachement bas.

Ces observations descriptives sont confirmées par les analyses bayésiennes. Nous avons effectué un codage centré des prédicteurs, l'attachement ( -1 pour l'attachement bas, 1 pour l'attachement haut) et la longueur avec la condition «Court» comme référence (-1 pour la condition «Court», 1 pour les autres). L'analyse confirme une probabilité de plus de $95 \%$ d'un effet de la hauteur de l'attachement ( $\beta$ hat $=5.16,95 \% \mathrm{CrI}=[3.83,6.6], \mathrm{P}(\beta)<0=0.95)$ et d'une interaction de la hauteur de l'attachement avec la longueur (interaction hauteur pour « Court» vs. 
« Moyen », $\beta$ hat $=-1.24,95 \% \mathrm{CrI}=[-2.33,-0.1], \mathrm{P}(\beta)<0=0.95$; interaction hauteur d'attachement et différence « Court » - « Long » : $\beta$ hat $=-3.14,95 \% \mathrm{CrI}=[-4.29,-2]$, $\mathrm{P}(\beta)<0=0.95)$. Il y a aussi un effet de longueur, qui signifie un désavantage fort pour les phrases plus longues (différence «Court»- «Moyen»: $\beta$ hat $=0.75,95 \%$ $\mathrm{CrI}=[0.12,1.37], \mathrm{P}(\beta)<0=0.95$; différence « Court » - «Long » : $\beta$ hat $=1.75,95 \%$ $\mathrm{CrI}=[1.17,2.37], \mathrm{P}(\beta)<0=0.95)$.

Figure 3a. Résultats de l'expérience sur le français

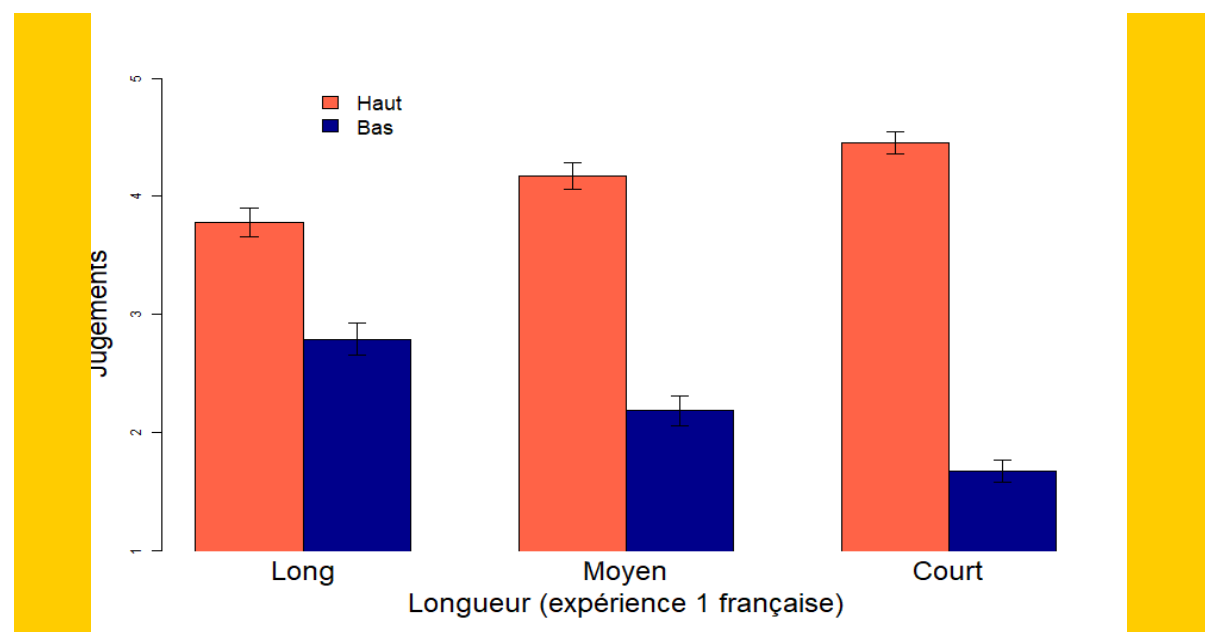

La figure $3 \mathrm{~b}$ montre les résultats de l'expérience sur l'anglais avec la même préférence pour un attachement haut et une interaction entre la hauteur de l'attachement et la longueur du premier conjoint. 
Figure 3b. Résultats de l'expérience sur l'anglais

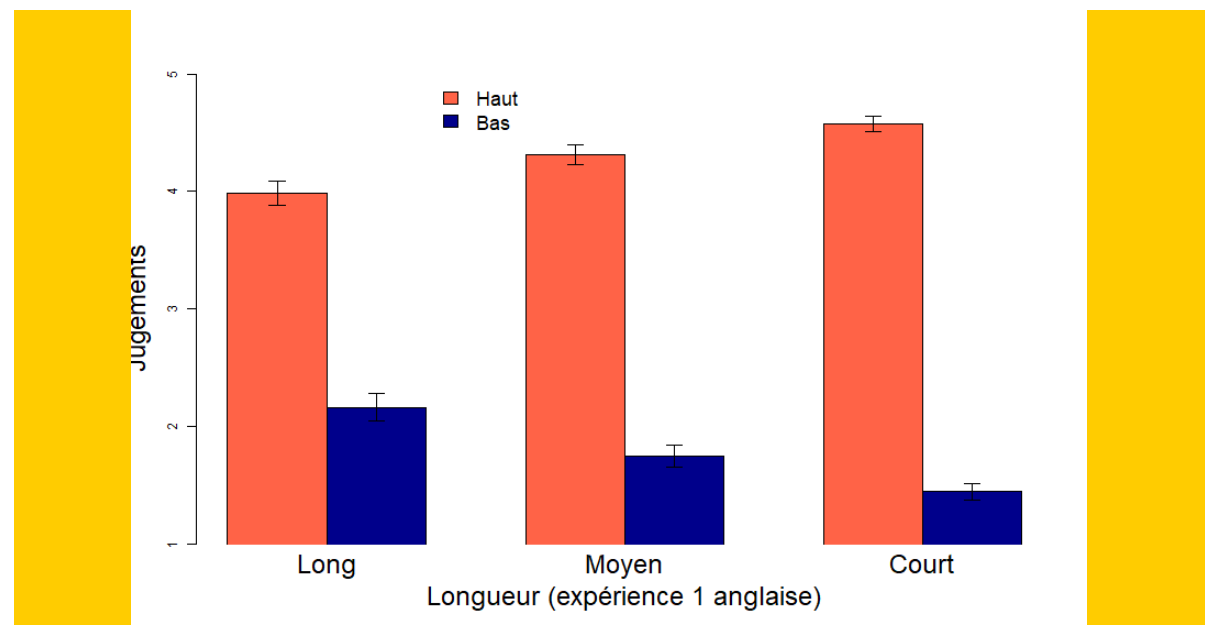

L'analyse montre plusieurs effets avec une probabilité au-delà de 95\%: l'effet de la hauteur $(\beta$ hat $=7.49,95 \% \mathrm{CrI}=[6.07,9.03], \mathrm{P}(\beta)<0=0.95)$; l'interaction hauteur d'attachement et différence «Court » - « Moyen » $(\beta$ hat $=-1.76,95 \% \mathrm{CrI}=[-2.79$, 0.73], $\mathrm{P}(\beta)<0=0.95)$; interaction hauteur d'attachement et différence "Court »«Long » ( $\beta$ hat $=-3.38,95 \% \mathrm{CrI}=[-4.89,-1.86], \mathrm{P}(\beta)<0=0.95)$ et effets de longueur, à savoir différence «Court» - « Moyen » $(\beta$ hat $=0.96,95 \% \quad \mathrm{CrI}=[0.33,1.6]$, $\mathrm{P}(\beta)<0=0.95)$; différence « Court » - « Long » $(\beta$ hat $=1.89,95 \% \mathrm{CrI}=[1.07,2.73]$, $\mathrm{P}(\beta)<0=0.95)$.

\subsection{Discussion}

Les résultats pour les variantes française et anglaise de l'expérience 1 confirment nos prédictions. Alors que les participants préféraient généralement un attachement haut, la longueur du premier conjoint modulait la préférence de telle sorte qu'un attachement bas devenait plus acceptable avec un premier conjoint plus long. Cependant, on pourrait avancer que la tâche de double jugement que nous avons utilisée dans cette expérience peut avoir amené les participants à considérer consciemment les deux alternatives et donc à appliquer des jugements plus métalinguistiques. Nous avons donc mené une deuxième expérience où les participants n'avaient à juger qu'une seule interprétation, un paradigme plus proche d'une expérience typique de jugement d'acceptabilité.

\section{DEUXIEME EXPERIENCE}

\subsection{Protocole expérimental}

Pour pallier les problèmes causés par la nature du paradigme de la première expérience, un paradigme plus classique de jugement d'acceptabilité (unique) est utilisé. En imposant une seule interprétation de la phrase relative par item, nous réduisons la probabilité d'un biais métalinguistique sur le jugement. 


\subsection{Matériels}

Pour la version française de l'expérience, les phrases de la première expérience ont été réutilisées : deux SN coordonnés suivis d'une phrase relative et puis d'un syntagme verbal. La variable manipulée est la longueur du premier SN. Ce dernier est allongé pour la condition longue à l'aide de deux adjectifs ou un adjectif et un syntagme prépositionnel. Nous avons enlevé la variante « Moyenne » de la première expérience pour alléger l'expérience. La deuxième variable manipulée est l'attachement: un attachement haut (sur la coordination des deux SN) ou un attachement bas (uniquement sur le deuxième $\mathrm{SN}$ ). Une deuxième phrase présentée après la première permet de forcer l'interprétation de la phrase souhaitée pour le jugement. Les participants sont invités à juger cette deuxième phrase. Un item de plus a été créé pour atteindre un total de 16 items, 4 items par condition (soit 64 phrases). Les items expérimentaux étaient mélangés avec 80 distracteurs pour une durée totale de l'expérience d'environ 20 minutes. Les items ont été répartis sur quatre listes en suivant le paradigme du carré latin comme pour l'expérience 1. Les items et les distracteurs ont été présentés dans un ordre aléatoire différent pour chaque participant.

Tableau 3. Exemple d'un item français dans les quatre conditions

\begin{tabular}{|l|l|l|}
\hline & Attachement haut & Attachement bas \\
\hline Court & $\begin{array}{l}\text { Les assiettes et les fourchettes qui se } \\
\text { trouvent sur l'étagère sont rayées. } \\
\text { Les assiettes et les fourchettes se } \\
\text { trouvent sur l'étagère. }\end{array}$ & $\begin{array}{l}\text { Les assiettes et les fourchettes qui se } \\
\text { trouvent sur l'étagère sont rayées. } \\
\text { Seules les fourchettes se trouvent sur } \\
\text { l'étagère. }\end{array}$ \\
\hline Long & $\begin{array}{l}\text { Les assiettes à couscous ovales et les } \\
\text { fourchettes qui se trouvent sur } \\
\text { l'étagère sont rayées. } \\
\text { Les assiettes et les fourchettes se } \\
\text { trouvent sur l'étagère. }\end{array}$ & $\begin{array}{l}\text { Les assiettes à couscous ovales et les } \\
\text { fourchettes qui se trouvent sur l'étagère } \\
\text { sont rayées. } \\
\text { Seules les fourchettes se trouvent sur } \\
\text { l'étagère. }\end{array}$ \\
\hline
\end{tabular}

Dans la version anglaise de l'expérience, les phrases conçues en français ont été traduites le plus fidèlement possible. Les mêmes conditions sont gardées : "Court » et «Long », deux adjectifs ou un adjectif et un syntagme prépositionnel sont ajoutés au SN pour obtenir la deuxième condition. 16 items ont été utilisés aussi pour cette expérience (soit 64 phrases).

Tableau 4. Exemple d'un item anglais dans les quatre conditions

\begin{tabular}{|l|l|l|}
\hline & Attachement haut & Attachement bas \\
\hline Court & $\begin{array}{l}\text { The plates and the forks that are on } \\
\text { the shelf are scratched. } \\
\text { Plates and forks are on the shelf. }\end{array}$ & $\begin{array}{l}\text { The plates and the forks that are on the } \\
\text { shelf are scratched. } \\
\text { Only the forks are on the shelf. }\end{array}$ \\
\hline
\end{tabular}




\begin{tabular}{|l|l|l|}
\hline Long & $\begin{array}{l}\text { The oval couscous plates and the } \\
\text { forks that are on the shelf are } \\
\text { scratched. } \\
\text { Plates and forks are on the shelf. }\end{array}$ & $\begin{array}{l}\text { The oval couscous plates and the forks } \\
\text { that are on the shelf are scratched. } \\
\text { Only the forks are on the shelf. }\end{array}$ \\
\hline
\end{tabular}

\subsection{Déroulement}

L'expérience est hébergée sur Ibex Farm (Drummond et al. 2016). Après avoir lu les instructions, avoir donné des informations sur leur âge, leur sexe et leur langue maternelle, et leur consentement de participer volontairement à cette expérience, l'expérience commençait avec trois items d'entraînement. Pour chaque phrase, les participants devaient déterminer l'acceptabilité de l'attachement de la relative (la deuxième phrase), et ceci sur une échelle de 1 (pas du tout acceptable) à 5 (tout à fait acceptable).

\subsection{Participants}

Pour la version française de l'expérience, 53 adultes $^{7}$ (locuteurs natifs du français) ont été recrutés sur les plate-formes RISC (https://www.risc.cnrs.fr) et Prolific (https://www.prolific.co/). Les participants sur Prolific ont reçu une compensation de $1.50 £$. La moyenne d'âge des participants est de 30.98 ans (minimum : 20 ; maximum : 68) pour 32 femmes et 21 hommes.

Pour la version anglaise de l'expérience, 47 adultes (locuteurs natifs de l'anglais) ont participé à cette expérience. Ils ont été recrutés via Amazon Mechanical Turk et reçu une compensation de $1.50 \$$. La moyenne d'âge des participants est de 33.7 ans (minimum : 18 ; maximum : 69) pour 23 femmes et 24 hommes.

\subsection{Résultats}

Nous attendons généralement une préférence pour l'attachement haut qui sera modulée par la longueur du premier SN. Nous attendons que l'attachement haut soit plus acceptable en général mais surtout pour un premier SN court. L'attachement bas devrait être plus acceptable avec un premier SN long qu'avec un premier SN court.

Pour la version française de l'expérience, nous retrouvons la préférence pour l'attachement haut, ainsi que l'interaction entre la longueur du premier conjoint et l'attachement. L'attachement bas devient plus acceptable avec un premier conjoint plus long.

3 personnes ont été exclues car le français n’était pas leur langue maternelle. 
Figure 4a. Résultats de l'expérience sur le français

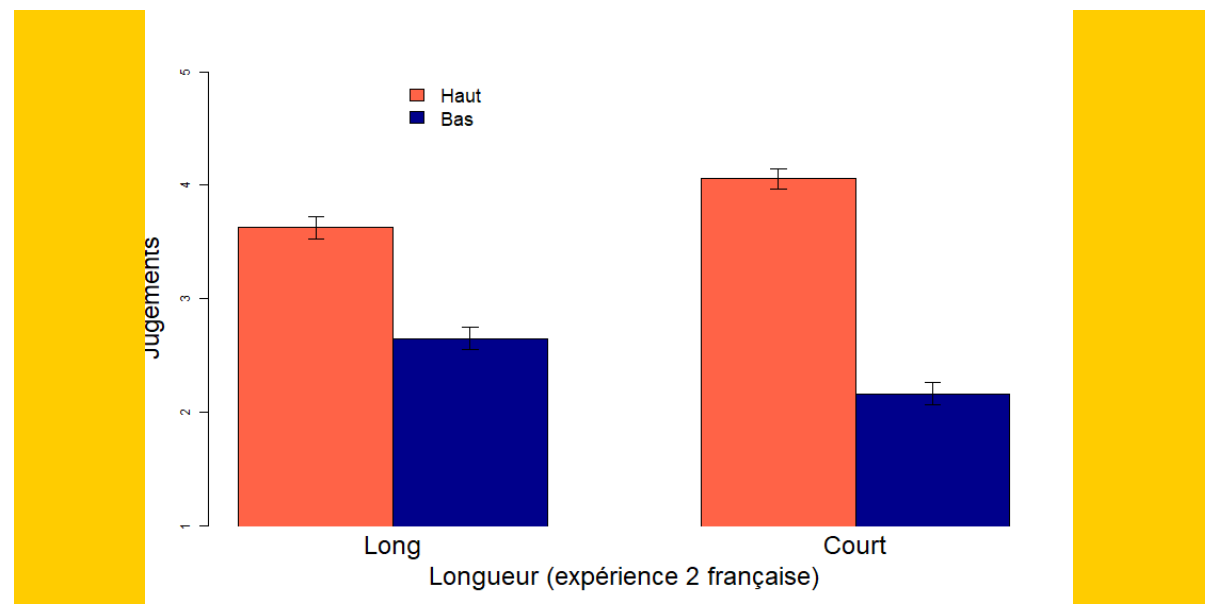

Pour les modèles bayésiens, les conditions de longueur et de hauteur ont été centrées et codées comme suit : long $=1$, court $=-1$; haut $=1$, bas $=-1$. L'analyse a retourné deux effets avec une probabilité au-dessus de $95 \%$, celui de la hauteur d'attachement $(\beta h a t=1.61,95 \% \mathrm{CrI}=[1.08,2.16], \mathrm{P}(\beta)<0=0.95)$ et l'interaction entre l'effet de longueur et l'effet de hauteur d'attachement ( $\beta$ hat $=-0.49,95 \% \mathrm{CrI}=[-0.85$, $-0.12], \mathrm{P}(\beta)<0=0.95)$.

Pour l'expérience en anglais, comme pour l'expérience en français, nous avons trouvé une forte probabilité pour un effet d'attachement avec une préférence pour l'attachement haut ( $\beta$ hat $=1.22,95 \% \mathrm{CrI}=[08,1.68], \mathrm{P}(\beta)<0=0.95)$, ainsi qu'une probabilité de $95 \%$ pour une interaction entre la longueur du premier conjoint et la hauteur d'attachement $(\beta$ hat $=-0.38,95 \% \mathrm{CrI}=[-0.64,-0.14], \mathrm{P}(\beta)<0=0.95)$. La préférence pour l'attachement haut est moins forte quand le premier conjoint est plus long.

Figure 4b. Résultats de l'expérience sur l'anglais
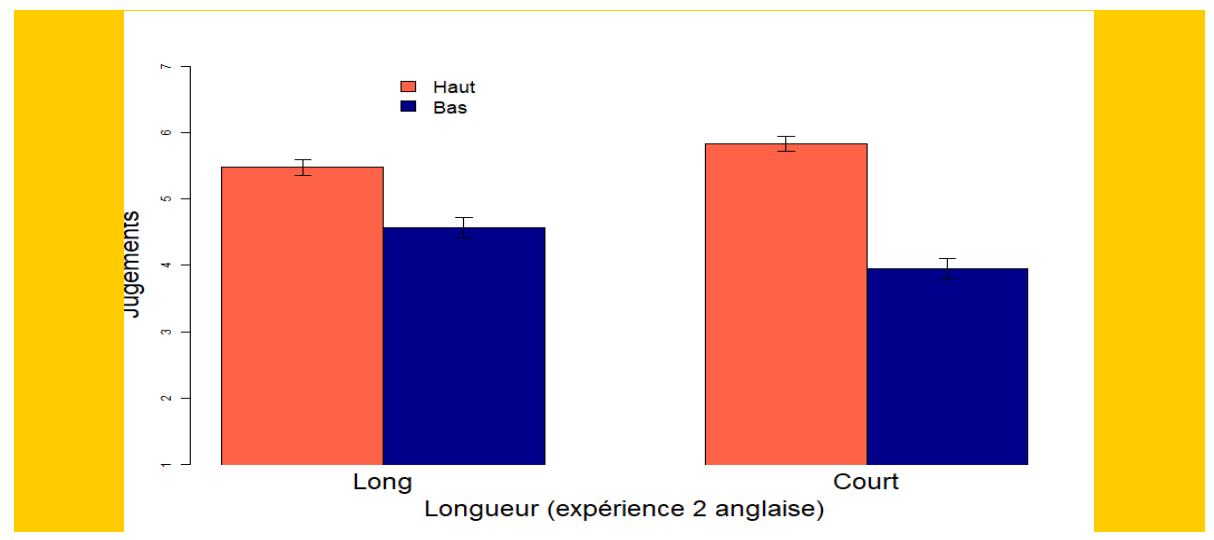


\subsection{Discussion}

L'expérience 2 confirme les résultats de l'expérience 1 tout en utilisant un paradigme expérimental plus traditionnel. Un attachement haut de la phrase relative est préféré comme auparavant dans les deux langues, mais un attachement bas devient plus acceptable avec un premier conjoint plus long.

\section{Discussion GENERALE}

Dans les deux langues et pour les deux paradigmes expérimentaux, nous constatons que les préférences d'attachement d'une phrase relative sont modifiées selon la longueur du premier SN coordonné. Plus ce syntagme est long, plus l'attachement de la phrase relative au deuxième SN devient acceptable. Il est important de noter que ce phénomène reste assez faible comparé à la préférence d'attachement haut largement observée à travers les deux langues. En revanche, il est très robuste, car il est observé et reproduit dans les quatre expériences. Nous avons donc suffisamment de raisons pour croire à l'existence de ce phénomène. Nos résultats sont compatibles avec les prédictions du principe des SSS même si la longueur du premier conjoint ne change pas complètement la préférence d'attachement. Quand le premier conjoint est assez long (4), l'attachement bas de la phrase relative rend une structure plus équilibrée que lorsque le première conjoint est court (5).

4. (Les assiettes à couscous ovales) et (les fourchettes qui se trouvent sur l'étagère) sont rayées.

5. (Les assiettes) et (les fourchettes qui se trouvent sur l'étagère) sont rayées.

Cependant, le principe des SSS porte ici sur une question de longueur, mais d'autres facteurs, comme par exemple l'équilibre entre poids sémantique et/ou pragmatique, pourraient intervenir (Hemforth et al. 2013). Lesquels de ces aspects jouent dans cette question de l'ambiguïté d'attachement des phrases relatives à deux SN coordonnés? En fait, le changement de la longueur du premier SN va de pair avec une variation de complexité syntaxique, sémantique et pragmatique. Hemforth et al. (2013) montrent qu'en français et en allemand la simple augmentation du nombre de syllabes ne change pas la préférence d'attachement des syntagmes prépositionnels à un SN complexe. L'ajout de contenu pragmatique, la charge informationnelle (Almor 1999), est en fait le facteur qui va avoir des effets sur la décision d'attachement.

Toutefois, dans nos expériences, nous ne distinguons pas pour l'instant la longueur et la complexité à ces niveaux, puisque cela demande des recherches plus approfondies. Cela signifie que, si nos résultats sont certainement compatibles avec une explication prosodique, d'autres études sont nécessaires pour mieux distinguer les effets de la prosodie de ceux de la complexité syntaxique ou sémantique ou de la charge informationnelle. 


\section{Références}

Almor A. (1999), «Noun-phrase anaphora and focus: The Informational Load Hypothesis ", Psychological Review 106, 748-765.

Baccino T., De Vincenzi M. \& Job R. (2000), « Cross-linguistic studies of the Late Closure Strategy: French and Italian ", in J. Flores d'Arcais (éd), Architectures and Mechanisms for Language Processing, Amsterdam, Elsevier, 25-34.

BEACH C. M. (1991), « The interpretation of prosodic patterns at points of syntactic structure ambiguity : Evidence for cue trading relations», Journal of Memory and Language 30(6), 644-663.

Bard E. G. \& Aylett M. P. (1999), «The dissociation of deaccenting, givenness, and syntactic role in spontaneous speech », ICPhS-14, 1753-1756.

Bard E. G., Anderson A. H., Sotillo C., Aylett M., Doherty-Sneddon G. \& Newlands A. (2000), "Controlling the intelligibility of referring expressions in dialogue », Journal of Memory and Language 42 (1), 1-22.

Barr D. J., LeVy R., Scheepers C. \& Tily H. J. (2013), «Random effects structure for confirmatory hypothesis testing: Keep it maximal », Journal of Memory and Language 68(3), 255-278.

BÜRKNER P. C. (2018), «Advanced Bayesian multilevel modeling with the R package brms », The R Journal 10(1), 395-411.

CARLson K., Frazier L. \& Clifton C. (2009), « How prosody constrains comprehension: A limited effect of prosodic packaging », Lingua 119(7), 1066-1082.

Cooper W. E. \& PAccia-CoOper J. (1980), Syntax and Speech. Harvard University Press.

Cuetos F. \& Mitchell D. C. (1988), "Cross-linguistic differences in parsing: Restrictions on the use of the Late Closure strategy in Spanish », Cognition 30(1), 73-105.

Drummond A., von der Malsburg T., Erlewine M. Y. \& Vafaie M. (2016), Ibex farm. [consulté le 15-12-2020 , disponible sur https:/github.com/addrummond/ibex/blob/master/docs/manual.md

Fodor J. D. (1998), « Learning to Parse? », Journal of Psycholinguistic Research 27(2), 285319.

FODOR J. D. (2002), "Psycholinguistics cannot escape prosody», in B. Bel \& I. Marlien (éds.), Speech Prosody 2002 : Proceedings, 83-88.

FRAZIER L. (1987), "Sentence processing: A tutorial review», in M. Coltheart (éd), The psychology of reading. Attention and performance 12, Hove-London-Hillsdale, Lawrence Erlbaum, 559-586.

FrAZIER L. \& Fodor J. D. (1978), « The Sausage Machine: A new two-stage parsing model », Cognition 6 (4), 291-325.

Frazier L. \& Clifton C., Jr. (1996), Construal, Cambridge, MA, MIT Press.

Gee J. P. \& Grosjean F. (1983), « Performance structures: A psycholinguistic and linguistic appraisal », Cognitive Psychology 15(4), 411-458.

Gibson, T., Pearlmutter, N., Canseco-Gonzales, E. \& Hickok, G. (1996), « Recency preference in the human sentence processing mechanism », Cognition 59, 23-59.

Gilboy E. \& SoPENA M. (1996), «Segmentation effects in the processing of complex NPs with relative clauses », in M. Carreiras, J. E. Garcia-Albea \& N. Sebastian-Galles (éds), Language processing in Spanish. Mahwah N. J., Lawrence Erlbaum Associates.

GRILlo N. \& COSTA J. (2014), « A novel argument for the universality of parsing principles », Cognition 133, 156-187. 
IGOA J. M. (1995), «Parsing decisions and the construal hypothesis: Attachment preferences in primary phrases in Spanish ", Paper presented at the Second Symposium on Psycholinguistics, Tarragona, Spain.

Hemforth B., Konieczny L. \& Scheepers C. (2000), « Syntactic attachment and anaphor resolution: The two sides of relative clause attachment », in M. Crocker, M. Pickering, \& C. Clifton, jr. (éds.), Architectures and mechanisms for language processing, Cambridge, UK, Cambridge University Press, 259-282.

Hemforth B. (2013), «Experimental Linguistics », in M. Aronoff (éd), Oxford Online Bibliographies, New York, Oxford University Press. [consulté le 16-12-2020, https://www.oxfordbibliographies.com ; DOI : 10.1093/OBO/9780199772810-0112]

Hemforth B., Colonna S., Petrone C. \& D'Imperio M. (2013), « Length matters: Informational load in ambiguity resolution », Discours 12. [consulté le 16-122020, http://discours.revues.org/8780 ; DOI : https://doi.org/10.4000/discours.8780]

Hemforth B., Mertins B. \& Fabricius Hansen C. (2014), Psycholinguistic approaches to meaning and understanding across languages. Studies in Theoretical Psycholinguistics, Dordrecht, Springer.

Hemforth B., Fernandez S., Clifton C., Jr., Frazier L., Konieczny L. \& Walter M. (2015), « Relative clause attachment in German, English, Spanish and French: Effects of position and length », Lingua 166, 43-64.

Inoue A. \& FodoR J. D. (1995), « Information-paced parsing of Japanese », in R. Mazuka \& N. Nagai (éds.), Japanese Sentence Processing. Hillsdale, Lawrence Erlbaum, 9-62.

JuN S.-A. (2003), "Prosodic phrasing and attachment preferences", Journal of Psycholinguistic Research 32 (2), 219-249.

KonieCZNy L. \& HEMFORTH B. (2000), « Modifier attachment in German: Relative clauses and prepositional phrases ", in A. Kennedy, R. Radach, D. Heller \& J. Pynte (éds.), Reading as a Perceptual Process. Amsterdam - Oxford, Elsevier, 517-527.

Kubozono H. (1993), The organization of Japanese prosody, (Studies in Japanese Linguistics 2.), Tokyo, Kurosio Publishers.

Michelas A. \& D'ImPerio M. (2015), « Prosodic boundary strength guides syntactic parsing of French utterances », Laboratory Phonology 6(1), 119-146.

MonNin P. \& Grosjean F. (1993), «Les structures de performance en français: caractérisation et prédiction ", L'Année Psychologique 9, 9-30.

Pozniak C., Hemforth B., Haendler Y., SAnti A. \& Grill N. (2019), « Seeing events vs. entities: The processing advantage of Pseudo Relatives over Relative Clauses », Journal of Memory and Language 107, 128-151.

Pynte J. \& Colonna S. (2000), « Decoupling syntactic parsing from visual inspection: The case of relative clause attachment in French », in A. Kennedy, R. Radach, D. Heller \& J. Pynte (Eds.), Reading as a perceptual process, Oxford, Elsevier, 529-547.

Schafer A, Speer S., Warren P. \& White D. (2000), «Intonational disambiguation in sentence production and comprehension», Journal of Psycholinguistic Research 29(2), 169-182.

WANNER E. (1980), "The ATN and the sausage machine: Which one is baloney? », Cognition, 8 (2), 209-225.

Zagar D., Pynte J. \& Rativeau S. (1997), « Evidence for early-closure attachment on firstpass reading times in French », Quarterly Journal of Experimental Psychology 50A, 421438. 